\title{
Editorial: New Piezoelectric Materials and Devices: Fabrication, Structures, and Applications
}

\author{
Chunlong $\mathrm{Fei}^{1 *}$, Lin Zhang ${ }^{2}$, Zeyu Chen ${ }^{3}$, Yang Yang ${ }^{4}$ and Jianguo $\mathrm{Ma}^{5}$ \\ ${ }^{1}$ School of Microelectronics, Xidian University, Xi'an, China, ${ }^{2}$ Media Lab, Massachusetts Institute of Technology (MIT), \\ Cambridge, MA, United States, ${ }^{3}$ College of Mechanical and Electrical Engineering, Central South University, Changsha, China, \\ ${ }^{4}$ Department of Mechanical Engineering, San Diego State University, San Diego, CA, United States, ${ }^{5}$ School of Instrumentation \\ and Optoelectronic Engineering, Beihang University, Beijing, China
}

Keywords: piezoelectric materials (PMs), piezoelectric devices (PZDs), fabrication, structures, applications, performance simulation

Editorial on the Research Topic

New Piezoelectric Materials and Devices: Fabrication, Structures, and Applications

\section{OPEN ACCESS}

Edited and reviewed by:

Weihua Li,

University of Wollongong

Wollongong, Australia

*Correspondence:

Chunlong Fei

clfei@xidian.edu.cn

Received: 29 November 2021

Accepted: 06 December 2021

Published: 05 January 2022

Citation:

Fei $C$, Zhang L, Chen Z, Yang $Y$ and

Ma J (2022) Editorial: New

Piezoelectric Materials and Devices:

Fabrication, Structures,

and Applications.

Front. Mater. 8:824345.

doi: 10.3389/fmats.2021.824345

\section{INTRODUCTION}

Piezoelectric materials and devices are fundamental to industrial and scientific applications such as non-destructive testing, acoustic tweezers, medical imaging, and ultrasound therapy. Typical piezoelectric materials include bulk ceramics, thin films, single crystals, polymers, composites, etc., which are used for a variety of devices such as ultrasound transducers, sensors, and actuators. This Research Topic of new piezoelectric materials and devices presents two reviews and eight original research articles that give insight into new piezoelectric materials, provide a basis for equivalent models of piezoelectric devices, and summarize and prospect the research of piezoelectric devices.

\section{PIEZOELECTRIC MATERIALS}

Piezoelectric materials have wide applications in energy conversion, sensors, drives, and frequency control. Chen et al. described rate-earth elements-doped piezoelectric materials that were categorized into ceramics, single crystals, and thin films. On this basis, the current research status and application prospects of those piezoelectric materials were summarized systematically, which can be used as the experimental design of piezoelectric materials to provide a level of theoretical reference.

Conventional lead-based piezoelectric materials for ultrasonic transducers are harmful to the human body and the environment. Consequently, Quan et al. reported the effect of $\mathrm{Sm}^{3+}$ modification for novel lead-free KNLN-BZ-BNT ceramics. When the temperature was raised from 30 to $180^{\circ} \mathrm{C}$, the thermal stability of the $d_{33}$ value decreased to less than $20 \%$. These results showed that $\mathrm{Sm}^{3+}$ modification can improve the properties of KNLN-BZ-BNT ceramics. Furthermore, the ceramics are suitable for further applications even under high temperatures.

Composite materials combine the advantages of piezoelectric materials and polymers to achieve high electromechanical coupling coefficients and low acoustic impedance. In this study, Han et al. proposed a 64-element ultrasonic phased-array transducer with a central frequency of $9 \mathrm{MHz}$ for 
intracardiac echocardiography. The phased array was fabricated based on 1-3 piezoelectric composites with a flexible circuit, and exhibited a high spatial resolution and imaging performance.

\section{DEVICES FABRICATION, STRUCTURES, AND APPLICATIONS}

Jun et al. introduced an ultrahigh frequency (UHF) ultrasonic transducer, and summarized the current research status from three aspects: material selection, focus design, and acoustic energy transmission matching. In addition, the authors also shared some views on the future development of UHF ultrasonic transducers. Shen et al. described the design and fabrication of ultrasonic transducers with different frequencies (12 and $20 \mathrm{MHz}$ ), different matching layers, and different cable lengths $(0.5-2.5 \mathrm{~m})$ to validate the effectiveness of the Leach model and the transmission line model. The results showed that in some applications, such as IVUS, the influence of the cable was not negligible, and even for high frequency transducers, different cable lengths had a significant effect on the transducer. The model of the sonotrode system was established to simulate the performance of two ultrasonic transducers and one sonotrode. Wang et al. presented the finite element model and the transfer matrix model for the sonotrode system. The vibration velocity ratio, resonance frequency, and amplitude ratio of the two models were compared. The proposed transfer matrix method is an effective method to simulate the dynamic performance of the ultrasonic welding electrode system, and provides a better basis for further optimization.

The surface acoustic wave (SAW) is an ultrasonic device which provides a wide range of applications with the use of an electronic system, including delay lines, filters, correlators, and DC to DC converters. Ma et al. explored the detailed fabrication process of a piezoelectric-on-silicon (POS) substrate, by using Smart-Cut technology, a $128^{\circ} \mathrm{Y}-\mathrm{X}$ LN POS substrate with a high-quality single-crystal LN film and low surface roughness was fabricated. Compared with the LN/Si structure, the BCB layer in the POS substrate could concentrate more SAW vibration in the piezoelectric thin film layer. Traditional radio frequency filters cannot meet the demands of miniaturization, high frequency operation, integration, and broadband capacity in newgeneration communication systems owing to their larger volumes. Jiang et al. first analyzed the harmonic characteristics of the FBAR and proposed the optimized structure of the FBAR by using the FEM method. Then, a $3.7 \mathrm{GHz}$ FBAR was fabricated through MEMS technology based on the optimized structural parameters. The simulation result was validated by comparing it to the FBAR test results. The proposed FBAR is perfectly compatible with currently available semiconductor technologies, allowing for device miniaturization and integration.

Guanbing et al. proposed a compact five-element integrated ultrasonic transducer, which was designed and manufactured for the inspection of defects in the split pins of control rod guide tubes in nuclear power plants. The compact $5 \mathrm{MHz}$ transducer has been proven to be capable of sustained operation in an underwater environment for 7 days, and can detect all the defects in the various zones of the split pin with a defect length of $10 \mathrm{~mm}$ and height sensitivity of $2 \mathrm{~mm}$. Due to strong background signals in vivo, it is difficult to image the molecules of a particular marker. Wang et al. developed a semi-ring array photoacoustic system for transient triple differential (TTD) imaging. PtOEP was used as the exogenous contrast agent and black ink was used to mimic the strong light absorption of melanin. According to the different delay TTD signals, the background signal was successfully removed by the TTD imaging principle. The ratio of the PtOEP signal to black ink signal increased to about 10 times. It is proved that the use of the TTD method to image phosphorescent materials with a strong background has great potential for molecular imaging in vivo.

In summary, these major research and review articles make this Research Topic a collection of new insights and perspectives on piezoelectric materials and devices. We thank all authors for their efforts and wish them success in continuously pushing their respective fields forward.

\section{AUTHOR CONTRIBUTIONS}

All authors listed have made a substantial, direct, and intellectual contribution to the work and approved it for publication.

Conflict of Interest: The authors declare that the research was conducted in the absence of any commercial or financial relationships that could be construed as a potential conflict of interest.

Publisher's Note: All claims expressed in this article are solely those of the authors and do not necessarily represent those of their affiliated organizations, or those of the publisher, the editors and the reviewers. Any product that may be evaluated in this article, or claim that may be made by its manufacturer, is not guaranteed or endorsed by the publisher.

Copyright $\odot 2022$ Fei, Zhang, Chen, Yang and Ma. This is an open-access article distributed under the terms of the Creative Commons Attribution License (CC BY). The use, distribution or reproduction in other forums is permitted, provided the original author(s) and the copyright owner(s) are credited and that the original publication in this journal is cited, in accordance with accepted academic practice. No use, distribution or reproduction is permitted which does not comply with these terms. 